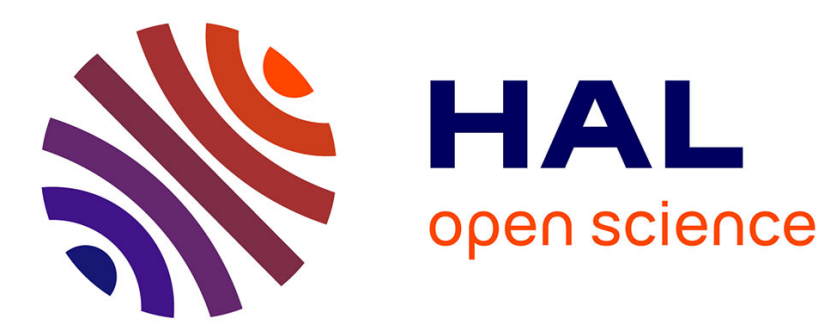

\title{
La cryomicroélectronique
}

Georges Kamarinos

\section{To cite this version:}

Georges Kamarinos. La cryomicroélectronique. Journal de Physique III, 1991, 1 (2), pp.283-294. 10.1051/jp3:1991123 . jpa-00248580

\section{HAL Id: jpa-00248580 https://hal.science/jpa-00248580}

Submitted on 1 Jan 1991

HAL is a multi-disciplinary open access archive for the deposit and dissemination of scientific research documents, whether they are published or not. The documents may come from teaching and research institutions in France or abroad, or from public or private research centers.
L'archive ouverte pluridisciplinaire HAL, est destinée au dépôt et à la diffusion de documents scientifiques de niveau recherche, publiés ou non, émanant des établissements d'enseignement et de recherche français ou étrangers, des laboratoires publics ou privés. 
Classification

Physics Abstracts

$85.30-85.40$

\section{La cryomicroélectronique}

\section{Georges Kamarinos}

Laboratoire de Physique des Composants à Semiconducteurs (URA CNRS 840), Institut National Polytechnique de Grenoble, ENSERG, BP 257, 38016 Grenoble Cedex, France

(Reçu le 6 mars 1990, accepté le 15 octobre 1990)

Résumé. - L'objet de la cryomicroélectroṇique est l'étude des Circuits Intégrés et des composants microélectroniques à des températures inférieures à $100 \mathrm{~K}$. Cet article de revue décrit très brièvement l'état d'avancement des connaissances relatives aux avantages et aux inconvénients des composants et Circuits Intégrés au Silicium fonctionnant à basse température. Ensuite on expose lés axes de recherche actuels; ils sont relatifs à deux approches : l'une, classique, est basée sur les matériaux utilisés actuellement dans la technologie de l'intégration à grande échelle; l'autre, novatrice, vise à utiliser les matériaux supraconducteurs à haute température critique.

Abstract. - The Integrated Circuits and the microelectronics devices working in temperatures lower than $100 \mathrm{~K}$ are studied by cryomicroelectronics. In this short review paper the development of this new branch of microelectronics is described. Particularly the advantages and the drawbacks of the cooling of integrated circuits and devices are listed. Then the current research axis are given. They correspond to two different approaches; the "classic" one which is based to the materials used in the present VLSI technology and the innovative way which aims at using new $\mathrm{H} T_{\mathrm{c}}$ superconductors.

\section{Introduction.}

Définitions ; caractéristiques techniques ; intérêt de refroidissement. - La cryomicroélectronique fait partie de la cryoélectronique ; son objet est l'étude des Circuits Intégrés (C.I.) (et des composants des C.I.) refroidis à des températures inférieures à $100 \mathrm{~K}$.

Dans l'état actuel de la technologie on peut distinguer deux voies dans la cryomicroélectronique :

a) la voie classique : il s'agit de la microélectronique refroidie utilisant les matériaux classiques et habituels (les semiconducteurs tel que le silicium ou l'AsGa, les diélectriques et les métaux de la microélectrónique fonctionnant autour de la température de $300 \mathrm{~K}$ ) ;

b) la voie utilisant totalement ou partiellement (cryomicroélectronique hybride) les nouveaux supraconducteurs à haute température critique.

La microélectronique est née vers 1960 de l'exigence à la fois économique et technique de fabriquer des systèmes :

- compacts

- peu volumineux 
- pas chers

- peu «énergivores »

- fonctionnant très rapidement.

Les caractéristiques techniques de la microélectronique sont essentiellement les suivantes :

- le faible coût

- la haute densité (ou la miniaturisation)

- la grande complexité (grand nombre de composants sur une puce)

- la faible dissipation d'énergie

- la grande fiabilité.

Le refroidissement permet d'augmenter la densité et la rapidité, tandis qu'il peut diminuer la dissipation d'énergie. On peut citer trois faits qui donnent naissance à la nécessité d'étudier la cryomicroélectronique :

- Il est évident (depuis maintenant 3-4 ans) que l'on améliore les caractéristiques des Circuits Intégrés au $\mathrm{Si}$ (ou à l'AsGa) si on les fait fonctionner à basses températures $(T<100 \mathrm{~K})[1-4]$.

- Depuis 6-7 ans, de nouveaux matériaux sont synthétisés pour répondre à l'exigence de miniaturisation (nouveaux diélectriques; nouveaux alliages métalliques, etc...). On constate que l'on ne connaît toujours pas l'effet du refroidissement sur le comportement électrique ou mécanique de ces mátériaux.

- Depuis 4 ans on se demande: «que faire des.nouveaux supraconducteurs à haute $T_{\mathrm{c}} ? »$.

Comme la microélectronique classique, la cryomicroélectronique doit se développer autour de deux grands axes : matériaux et dispositifs ; évidemment les activités de modélisation, simulation et C.A.O. reliant ces deux axes doivent aussi être importantes.

Les matériaux dont on doit étudier la technologie et les propriétés sont principalement : les isolants, les siliciures et les nouveaux supraconducteurs.

Les dispositifs que l'on doit étudier à basse température sont principalement les transistors MOS (TMOS) et les transistors bipolaires à hétérojonction.

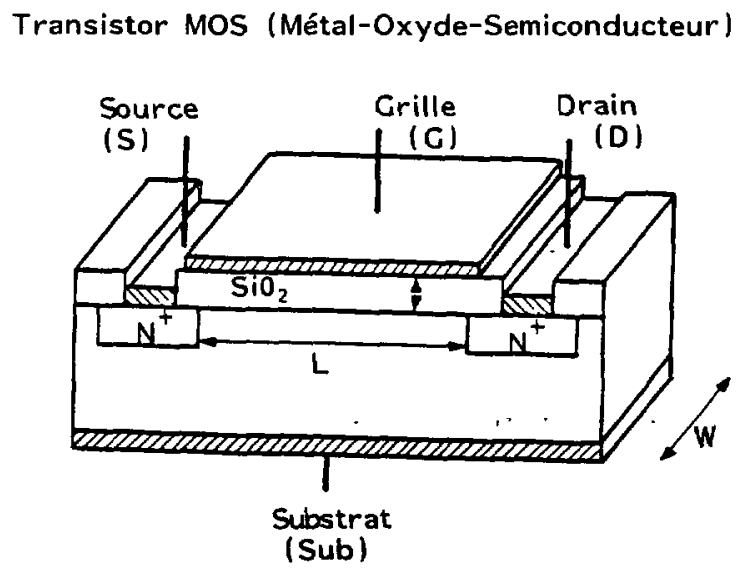

Fig. 1. - Le Transistor Métal-Oxyde-Semiconducteur.

[Metal-Oxide-Semiconductor Transistor.] 
La modélisation et la simulation électrique du fonctionnement des dispositifs des C.I., y compris la réponse en hautes fréquences des connexions refroidies, sont nécessaires.

Il est incontestable que la microélectronique au silicium va garder, pour une décennie au moins, une place plus que dominante dans les applications et par conséquent dans le marché des C.I.

C'est pour cela, et pour ne pas alourdir excessivement la contribution, que cet article ne s'occupera que des dispositifs et C.I. au silicium.

Les propriétés de ce semiconducteur à basses et très basses températures sont étudiées et connues depuis longtemps. La cryomicroélectronique au silicium ne peut donc s'articuler qu'autour du comportement du transistor MOS (TMOS) pour des raisons que l'on verra plus loin (Fig. 1).

\section{Le TMOS refroidi à $T \approx 77 \mathrm{~K}$.}

Avantages ; inconvénients ; intérêt de la descente à des températures très basses. - Plusieurs travaux de recherche ont déjà été consacrés au comportement du TMOS aux basses ou aux très basses températures [1-5].

\subsection{AVANTAGES DU REFROIDISSEMENT DU TMOS, ÉlÉMENT D'UN C.I.}

(i) La vitesse d'entraînement des porteurs libres de charge s'accroît. Ceci est dû à l'augmentation de la mobilité ( $\mu$ ) (Figs. 2 et 3 ) $[6,7]$

$$
v=\mu E ; \mu=q \tau / m
$$

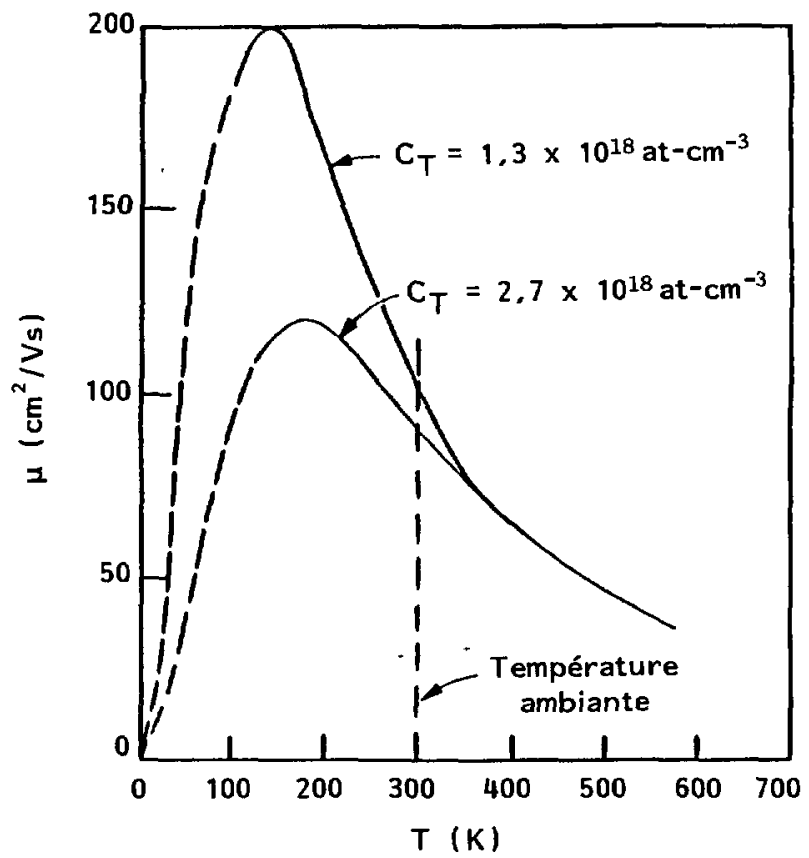

Fig. 2. - Variation de la mobilité des électrons en fonction de la température.

[Electron mobility variation vs. temperature (cf. A. S. Grove : Physique et Technologie des dispositifs à semiconducteurs, Ed. Dunod, Paris, 1971).] 


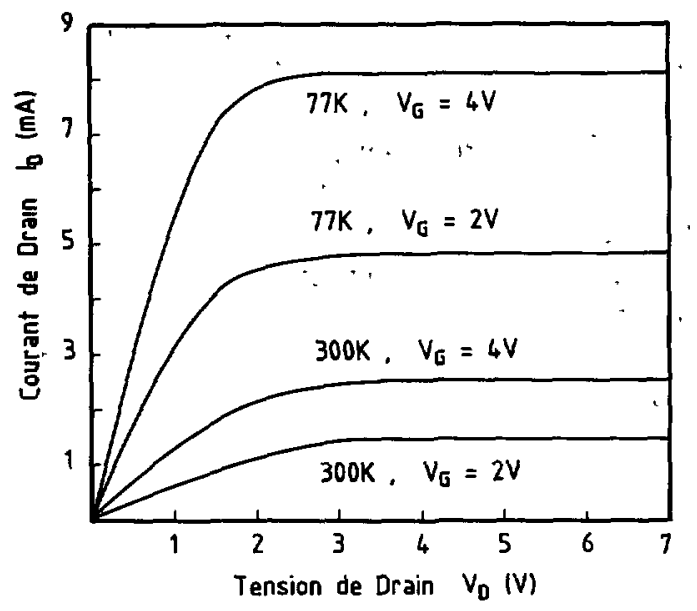

Fig. 3. - Caractéristiques électriques $I_{\mathrm{D}}$ (courant source-drain) en fonction de la polarisation de drain $V_{\mathrm{D}}$ pour plusieurs valeurs de la tension de grille $V_{\mathrm{G}}$. Caractéristiques données pour ${ }_{\mathrm{t}} 77 \mathrm{~K}$ et $300 \mathrm{~K}$.

[Electrical characteristics $I_{\mathrm{D}}, V_{\mathrm{D}}$ for various gate voltages $V_{\mathrm{G}}$ for two temperatures : $77 \mathrm{~K}$.and $300 \mathrm{~K}$.]

où $E$ est le champ électrique, $q$ la charge du porteur $\left( \pm 1,6 \times 10^{-19} \mathrm{C}\right), \tau$ le temps moyen de libre parcours et $m$ la masse effective du porteur (électron ou trou).

On constate aussi une augmentation significative ( $30 \%$ en passant de $300 \mathrm{~K}$ à $77 \mathrm{~K}$ ) de la vitesse de saturation (à hauts champs électriques) et de la vitesse "overshoot» (cas de l'AsGa) [8, 9].

On aboutit ainsi à une plus grande rapidité de fonctionnement.

Remarque. - Il ne faut pas oublier que la mobilité n'est pas une fonction monotone de $T$; elle passe par un maximum à une température $T$ dépendant du dopage $[6,7]$.

(ii) La pente $S$ en inversion faible du TMOS augmente $\cdot[10]$

$$
\frac{\partial \ln I_{\mathrm{D}}}{\partial V_{\mathrm{G}}}=S=\frac{C_{\mathrm{ox}}}{C_{\mathrm{ox}}+C_{\mathrm{D}}+C_{\mathrm{ss}}} \frac{q}{k T}
$$

où $I_{\mathrm{D}}$ est le courant source-drain, $V_{\mathrm{G}}$ la tension de grille, $k$ la constante de Boltzmann. $C_{\mathrm{ox}}, C_{\mathrm{D}}$ et $C_{\mathrm{ss}}$ sont respectivement les capacités dites d'oxyde, de la zone désertée et des états de surface [10].

On aboutit ainsi à des densités d'intégration supérieures et on pourrait aussi diminuer la tension d'alimentation $V_{\mathrm{D}}$ (actuellement $V_{\mathrm{D}}=5 \mathrm{~V}$ ) [11].

(iii) Les courants "de fuite" diminuent.

(iv) Le bruit thermique diminue; ceci plaide pour la diminution de $V_{\mathrm{D}}$. Mais le bruit thermique n'est qu'une composante du bruit total (bruit de diffusion + bruit de générationrecombinaison + bruit de grenaille) $[5,12-14]$.

Ainsi dans l'état actuel des recherches on peut dire que l'abaissement de la température ne donne pas naissance à des sources de bruit inattendues. Mais en général les dispositifs refroidis deviennent plus bruyants et les effets qui entrent en jeu (gel des impuretés, ionisations, etc.) peuvent expliquer les «nouveaux » bruits en excès [15].

(v) La conductivité thermique $K$ augmente. Ceci autoriserait une densification du circuit. Mais il ne faut pas oublier que pour les semiconducteurs, qui ne suivent pas la loi de 


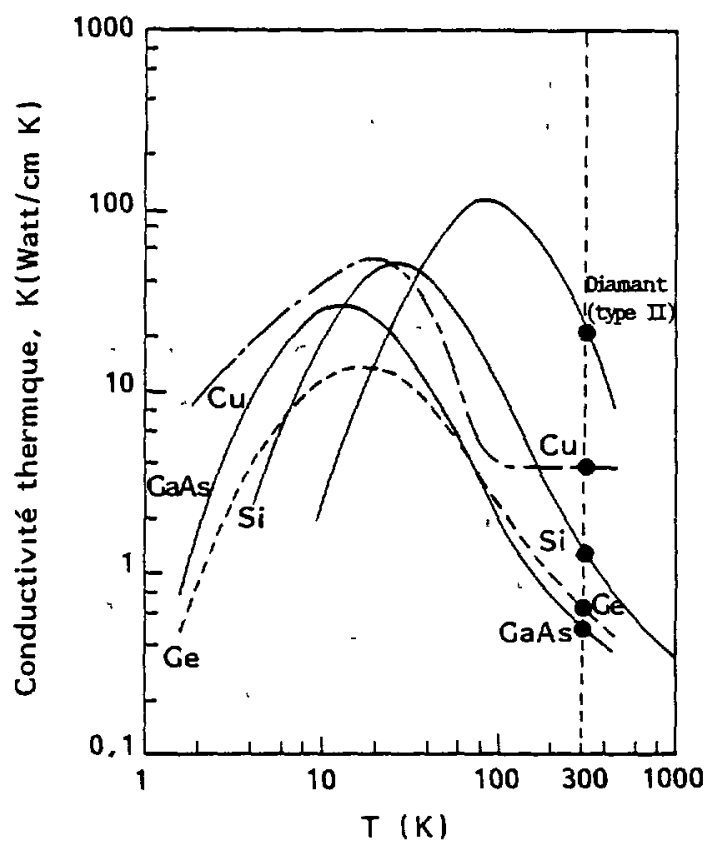

Fig. 4. - Variation de la conductivité thermique de quelques semiconducteurs usuels; comparaison avec le cuivre.

[Thermal conductivity vs. temperature for some usual semiconductors; the Copper curve is given for comparison (SZE : op. cit.).]

Widermann-Franz entre autres, $K$ n'est pas une fonction monotone de la température. Pour le silicium en particulier $K$ atteint un maximum autour de $30 \mathrm{~K}[6,16]$ (Fig. 4).

(vi) Le phénomène de verrouillage disparaît. Cet effet, connu sous le vocable «latch-up ", est dû au couplage des transistors n-TMOS et p-TMOS d'une cellule CMOS [17-19]; entre les sources de deux transistors un comportement de type "thyristor " $\mathrm{n}^{+} \mathrm{p} \mathrm{n}^{+} \mathrm{p}^{+}$s'installe et la cellule est court-circuitée. Cet effet disparaît rapidement avec la diminution des courants de fuite [17].

(vii) Les capacités de jonction diminuent (les zones de charge d'espace s'étalent). Par conséquent la fréquence de coupure $f_{\mathrm{c}}$ (rapidité) du TMOS augmente; ceci plaide aussi pour une densification du C.I.

(viii) La conductivité des interconnexions métalliques augmente. Ainsi la rapidité du C.I. se trouve augmentée et la dissipation de.chaleur diminuée.

(ix) L'effet d'électromigration diminue exponentiellement avec la température. Ceci est dû au fait que l'électromigration est un phénomène thermoactivé, répondant par conséquent à une loi d'Arrhenius. La quasi-disparition de l'électromigration avec l'abaissement de la température de fonctionnement augmente la durée de vie et par là, la fiabilité du C.I. et du système [20].

En ce qui concerne les caractéristiques $\left(I_{\mathrm{D}}, V_{\mathrm{D}}\right) V_{\mathrm{G}}$ d'un TMOS refroidi à $77 \mathrm{~K}$ on peut faire brièvement les remarques suivantes (Fig. 3 ) :

(i) à $77 \mathrm{~K}$ les courants de saturation sont supérieurs et les tensions $V_{\mathrm{D}}$ de saturation inférieures

(ii) la réduction à des géométries submicroniques modère les tendances ci-dessus [10]. 


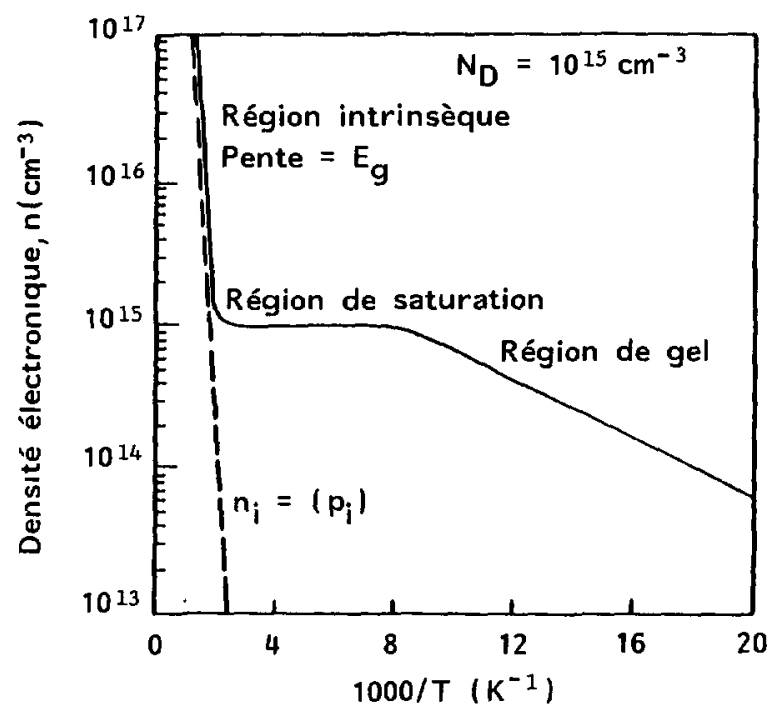

Fig. 5. - Densité d'électrons libres dans un semiconducteur en fonction de la température (silicium).

[Free Electron density in Silicon vs. temperature (cf. SZE : Physics of Semiconductor Devices, Ed. J. Wiley 1969).]

Ces comportements sont explicables après une analyse attentive et détaillée des effets de transport des charges libres dans le canal du TMOS. L'échauffement des électrons et des trous se déplaçant dans un champ électrique $\left(E>10^{4} \mathrm{~V} / \mathrm{m}\right)$ saturant leur vitesse (canaux microniques et submicroniques) joue un rôle essentiel $[10,21]$.

2.2 INCONVÉNIENTS DU REFROIDISSEMENT DU TMOS, ÉLÉMENT D'UN C.I. - Remarquons d'emblée que le dispositif de refroidissement à l'azote liquide ne semble plus constituer un inconvénient majeur. L'inconvénient principal, et peut être unique, du refroidissement est le vieillissement accéléré du TMOS entraînant la défaillance du C.I. et par conséquent la nonfiabilité du C.I. ou du système (Figs. 6-10) [10].

Par vieillissement on entend le glissement des caractéristiques (tension de seuil $V_{\mathrm{T}}$, transconductance $g_{\mathrm{m}}$, etc.) du TMOS en fonction de la durée de son fonctionnement. Le vieillissement est dû essentiellement à l'échauffement des porteurs sous des champs électriques élevés $\left(E>10^{4} \mathrm{~V} / \mathrm{m}\right)$. Il s'ensuit une détérioration de la zone de drain, ainsi qu'une injection et un piégeage des porteurs dans l'oxyde. C'est un inćonvénient majeur de la miniaturisation et de l'avènement de la microélectronique submicronique et par conséquent, il hante les filières VLSI. Son étude et la découverte des remèdes techniques constituent un important défi pour le développement des filières de la prochaine décennie ( $p$. ex. voir programme Européen JESSI). Malheureusement tout montre que le refroidissement aggrave cet effet.

Le vieillissement du TMOS concerne donc toute la microélectronique et pas seulement la cryomicroélectronique. On doit signaler aussi ici que les modèles existants dans les outils de simulation habituels sont caducs quand on veut étudier le TMOS refroidi [22]. Une modélisation spéciale est donc nécessaire, surtout quand on descend à des températures où l'on doit tenir compte des effets de quantification des états énergétiques de l'électron dans la couche d'inversion. 


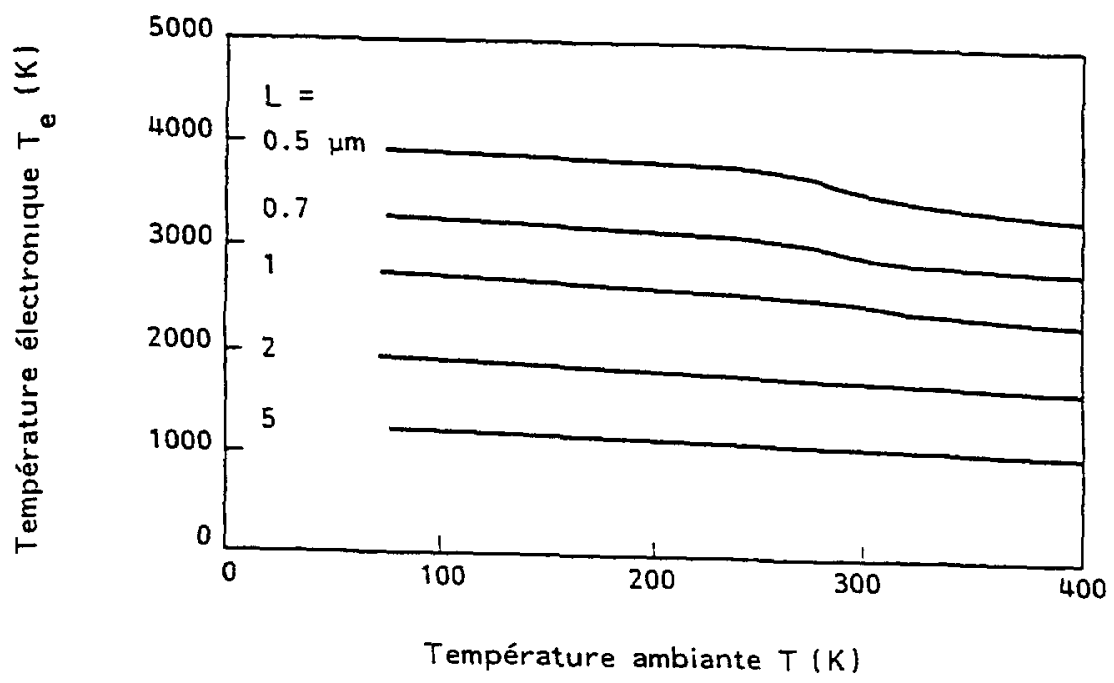

Fig. 6. - Température des électrons dans le canal d'un TMOS fonctionnant à diverses températures ambiantes : porteurs chauds.

[Free electron temperature into the channel of a MOST working at different temperatures: hot carriers.]

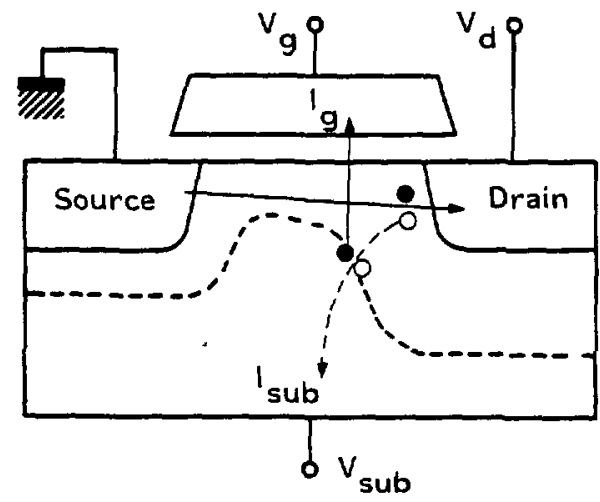

Fig. 7. - Vieillissement du TMOS : un cas d'injection de porteurs chauds dans l'oxyde de grille. [Ageing of a MOST : a case of injection of hot carriers into gate oxide.]

Remarquons aussi que les transistors bipolaires classiques deviennent inefficaces aux basses températures. En effet, du surdopage de l'émetteur par rapport à la base résulte une diminution $\Delta E_{\mathrm{g}}$ de la bande interdite, $E_{\mathrm{g}}$, du semiconducteur. Il s'ensuit une réduction de l'efficacité de l'émetteur et un gain $\beta$ diminuant exponentiellement avec la température

$$
\beta=\beta_{300} \exp \left(-\frac{\Delta E_{\mathrm{g}}}{k T}\right) \text { où } \beta_{300} \text { est le gain à } 300 \mathrm{~K} \text {. }
$$

L'utilisation des transistors bipolaires classiques est donc très compromise ; on recourt alors aux transistors bipolaires à hétérojonction (HBT) [23-25]. 


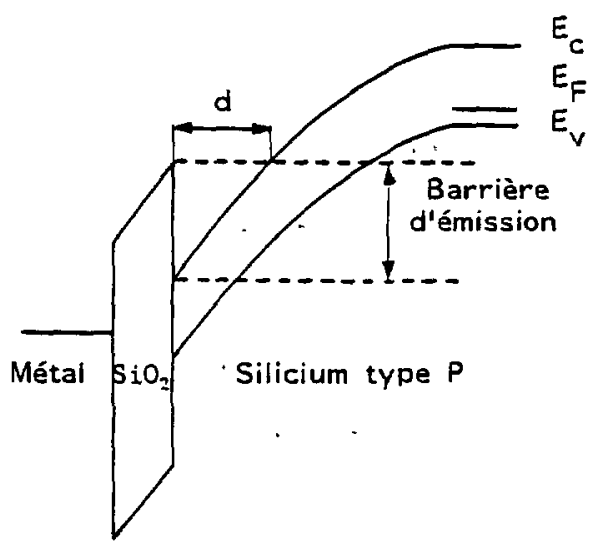

Fig. 8. - Vieillissement du TMOS : profil de bandes à l'interface $\mathrm{Si}_{-} \mathrm{SiO}_{2}$ à travers laquelle l'injection s'opère. Probabilité d'injection selon le modèle d'électron chanceux de Shockley $P_{\mathrm{n}_{\mathbf{y}}}=P_{0} \exp (-d / \lambda)$ où $\lambda$ est le libre parcours moyen du porteur dans l'oxyde (quelques dizaines d' $\AA$ ).

[Ageing of MOST: band profile in the $\mathrm{Si}-\mathrm{SiO}_{2}$ interface through which the injection of hot carriers occurs. Injection probability according the lucky-electron model : $P_{1 \mathrm{nj}}=P_{0} \exp (-d / \lambda)$ where $\lambda$ is the carrier mean free path in the gate oxide (some tens of $\AA$ ).]

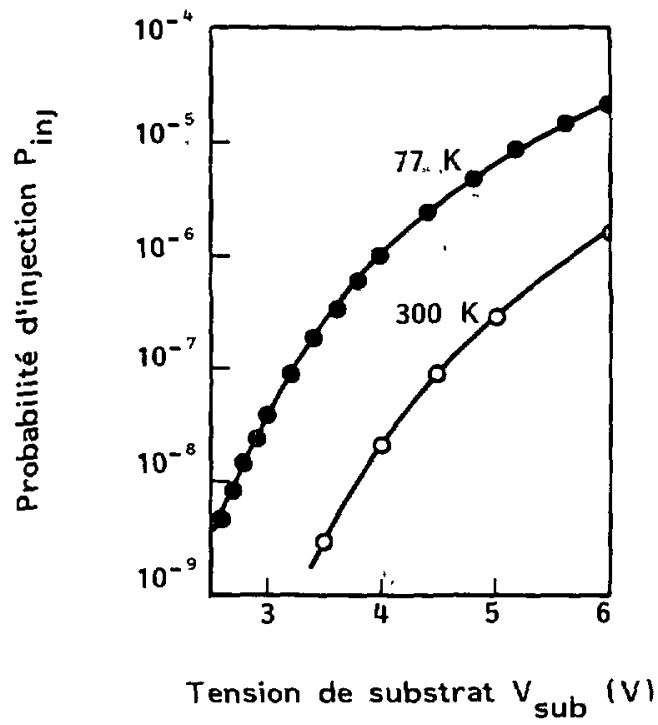

Fig. 9. - Vieillissement des TMOS : váriation de $P_{\mathrm{ln}\}}$ en fonction de la température.

[Ageing of MOST : variation of $P_{\mathrm{inj}}$ vs. temperature.]

Quand on descend encore plus bas en température $(4,2 \mathrm{~K}<\mathrm{T}<60 \mathrm{~K})$, les problèmes que l'on doit affronter sont :

- le gel des impuretés [5, 6, 26] (Fig. 5)

- l'ionisation par choc des impuretés ou par effet Poole-Frenkel [6-8, 27]

- la réduction éventuelle de la mobilité $[5-7,28-30]$ 


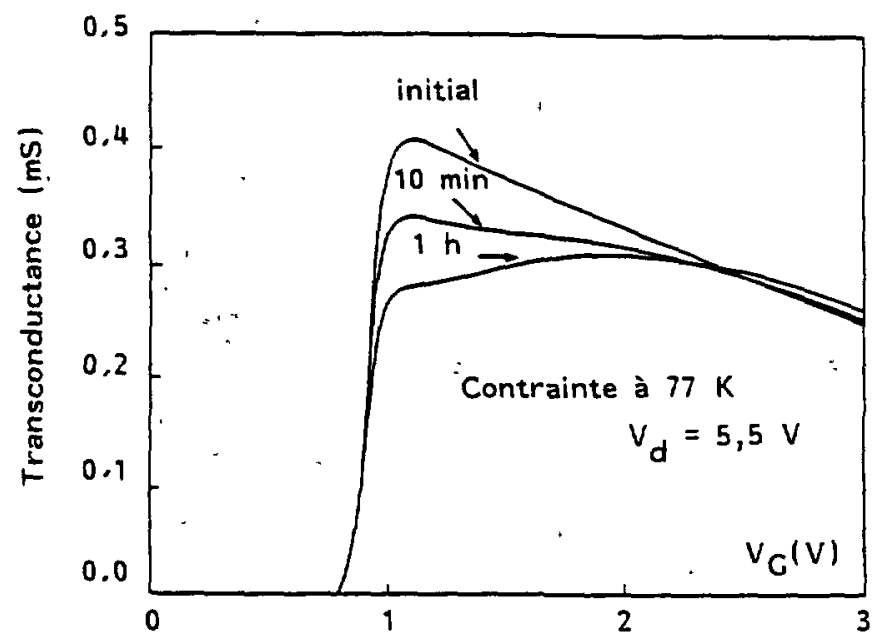

Fig. 10. - Vieillissement du TMOS : variation de la transconductance en fonction de $V_{\mathrm{G}}$. [Ageing of MOST : variation of the transconductance $v$ s. gate voltage $V_{\mathrm{G}}$.]

- la réduction éventuelle de la conductivité thermique $[6,16]$

- la quantification des niveaux énergétiques dans la couche d'inversion [31, 32]

- les instabilités électriques diverses

- l'augmentation de la résistance série $[33,34]$

- l'effet kink [35-37].

L'effet kink dû à une autopolarisation du transistor.MOS à la suite d'une ionisation près du drain (et l'apparition d'un courant de substrat), est présent dès que l'on s'approche de $30 \mathrm{~K}$ [38]. Il est présent, même à $T=300 \mathrm{~K}$ dans toutes les filières utilisant le silicium sur isolant $[39,40]$. (Ne pas oublier qu'à très basse température le substrat silicium devient isolant) (Fig. 11).

\section{Axes de recherche.}

Ce qui précède montre que la cryomicroélectronique au silicium traverse actuellement son étape d'évaluation. Ce șont les travaux de recherche en cours ou à engager qui préciseront définitivement l'intérêt et les limites de cette technique.

3.1 APPROCHE CLASSIQUE. - Elle est basée sur l'évaluation des matériaux à comportement «classique » et des composants intégrés dans une filière refroidie.

3.1.1 Matériaux. - Il s'agit des matériaux isolants $\left(\mathrm{SiO}_{2}\right.$, nitrure, oxynitrures), siliciures (WSi ${ }_{2}, \mathrm{TiSi}_{2}, \mathrm{CoSi}_{2}$, etc.) et métaux (à signaler le retour du cuivre).

Les thèmes de recherche seraient :

a) pour les isolants

- l'étude de la constante diélectrique complexe $\varepsilon=\varepsilon^{\prime}+j \varepsilon^{\prime \prime}$ en fonction de la température

- l'étude du claquage à haut champ électrique

- la caractérisation électronique (densité, état énergétique, propriétés de piégeage) des interfaces Isolant-Semiconducteur ou Isolant Isolant $_{2}$ et ceci en fonction et en corrélation avec les différents procédés techniques d'obtention des couches diélectriques (oxydation, nitruration, CVD, LPCVD, PECVD...). 


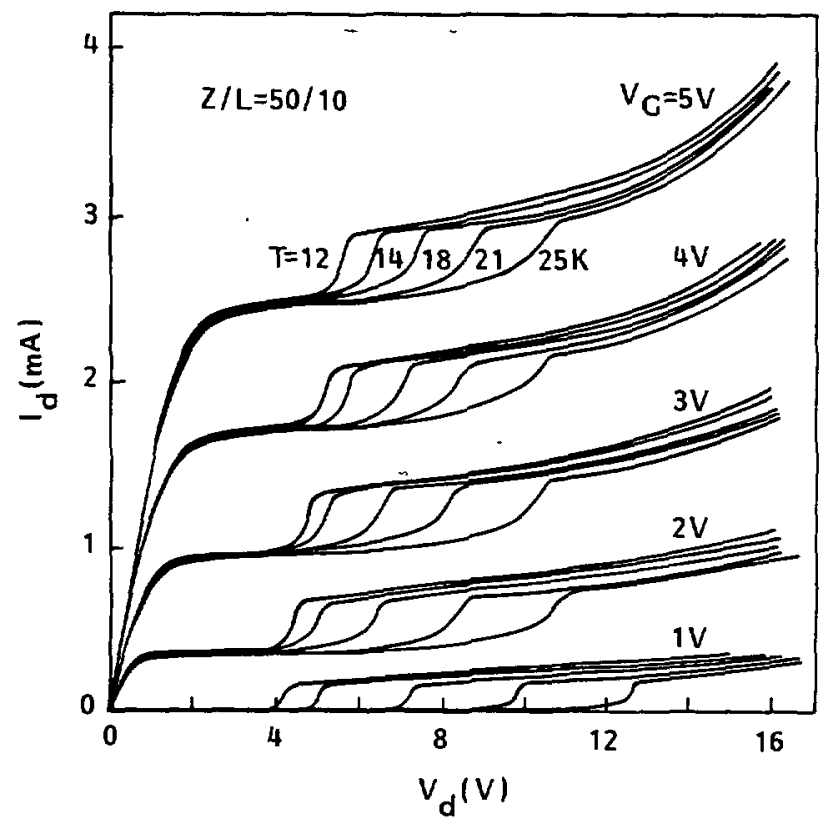

Fig. 11. - Effet kink.

[Kink effect.]

- l'étude des propriétés structurales et mécaniques à basses températures.

b) Pour les siliciures et les métaux (en couches minces) :

- l'étude de leurs propriétés électriques en fonction de la température ( $\sigma$, états d'interface, barrière de potentiel Schottky, etc.)

- l'étude de leurs propriétés structurales et mécaniques ( $p$ : ex. adhésion, coefficient de dilatation, etc.).

3.1.2 Les composants et les C.I. - Il s'agit essentiellement des TMOS/Si, éléments des C.I. (pour l'AsGa et les filières III-V les MESFET, les HBT, les HEMT sont les dispositifs à étudier). Les thèmes de recherche seraient :

- l'optimisation des procédés techniques de réalisation et des règles de dessin des C.I. CMOS ;

- l'effet des cycles thermiques;

- l'élaboration des modèles de fonctionnement à basse température ;

- la compréhension des transports (effet de gaz électronique bidimensionnel) dans les couches d'inversion;

- l'étude du vieillissement et l'élaboration des techniques le réduisant;

- l'analyse du bruit et des fluctuations électriques à basse température et plus particulièrement pour les filières submicroniques;

- l'analyse des effets quantiques émergeant à basse et surtout à très basse $(T<30 \mathrm{~K})$ température (localisation, quantification, ...);

- l'élaboration d'outils de simulation numérique du fonctionnement des C.I. (y compris la réponse en haute fréquence des interconnexions refroidies). 
3.2 APPROCHE NOVATRICE : FILIÈRES SUPRACONDUCTEURS. - Les nouveaux supraconducteurs à haute température critique peuvent être utilisés pour [41]:

(i) les interconnexions $[42,43]$

(ii) une électronique entièrement en supraconducteurs (utilisant les dispositifs actifs à effet Josephson p. ex. [44, 45])

(iii) une électronique hybride où seulement les parties actives sont en supraconducteurs $[42,45]$.

Le principal avantage de l'utilisation des nouveaux supraconducteurs serait une diminution considérable des résistances série et des résistances d'interconnexions; la rapidité donc serait augmentée et la puissance dissipée diminuée. Il en résulterait une plus haute densité d'intégration.

L'inconvénient actuel est qu'aucun problème technologique majeur n'est encore résolu.

De plus le choix du composé supraconducteur est difficile; en effet on doit :

- rendre compatibles les matériaux de la microélectronique classique $\left(\mathrm{Si}, \mathrm{SiO}_{2}, \mathrm{Al},.\right)$ avec les supraconducteurs;

- optimiser les techniques de dépôt et rendre reproductibles les propriétés des couches supraconductrices ;

- étudier les couches-tampon (buffer-layers);

- etc, .

\section{Conclusion.}

La cryomicroélectronique, branche de la microélectronique s'annonce prometteuse. Ses domaines d'application sont ceux de la microélectronique classique; déjà en électronique spatiale et pour des calculateurs rapides on utilise des C.I. refroidis.

Cependant les défis techniques sont nombreux. La recherche fondamentale et technologique peut être nourrie pendant plusieurs années des questions provenant de la cryomicroélectronique.

\section{Remerciements.}

L'auteur remercie Gérard Ghibaudo et Francis Balestra, qui animent au Laboratoire de Physique des Composants à Semiconducteurs, les recherches sur la cryomicroélectronique et avec qui il a eu de nombreuses discussions.

\section{Bibliographie}

[1] Numéro Spécial IEEE Trans. Electron. Devices ED-34 (1987).

[2] Rogers G., Solid State Electron. 11 (1968) 1079.

[3] Keyes R. W., Harris E. P., Konnerth K. L., Proc. IEEE 58 (1970) 1914.

[4] Sun J. Y. C., Taur Y., Dennard R. H., Klepner S. P., Wang L. K., IEDM, Tech. Dig. (1986) 236.

[5] Balestra F., Ghibaudo G., Hafez I. M., Kamarinos G., Physics of Semiconductor Devices, Proc. 5-th Int. Workshop (New-Delhi, 1989) p. 128.

[6] SzE S. M., Physics of Semiconductor Devices, Ed. J. Wiley (New-York) 1969.

[7] Grove A. S., Physique et Technologie des Dispositifs à Semiconducteurs, Ed. Dunod (Paris) 1971.

[8] Conwell E. M., High Field Transport in Semiconductors (Acad. Press, New-York) 1967.

[9] NAG B. R., Theory of Electrical Transport in Semiconductors (Pergamon Press, New-York) 1972. 
[10] ChiÊn NguYen-Duc, Etude du fonctionnement et du vieillissement du TMOS Submicronique à basse température, Thèse INPG Grenoble (1988).

[11] Sat-Halasz, Wordeman M., Kern D. P., Ganin E., Rishton S., Zicherman D. S., Schmid H., Polcari M. R., Ng N. Y., Restle P. J., Chang T. H. P., Dennard R. H., ieEe E.D.L. 8 (1987) 463.

[12] Hafez I. M., Ghibaudo G., Balestra F., J. Appl. Phys. 66 (1989) 2211.

[13] 'Adkins C., Koch R., J. Phys. C 15 (1982) 1829.

[14] Hendriks E., Zijlstra, J. Solid State Electron. 31 (1988) 1105.

[15] Workshop on Noise in Semiconductor Devices, Proc. Ed. ENSERG (LPCS, Grenoble) 1990.

[16] Drabble J. R., Goldsmitt H. J., The Thermal Conduction in Semiconductors (Pergamon Press, New-York) 1961.

[17] LeRoux Ch., Thèse, INSA Lyon (1988).

[18] Coppage F. N., Evans D. C., IEEE Trans. on Nucl. Sci. 24 (1977) 2226.

[19] Colett R., Digitàl Design 15 (1985) 62.

[20] Lloyd J. R., Pierce J., Levy R. A., Frieser R. G., Electromigration of Metals, Proc. Electron. Soc. 85-6 (Electroch. Soc., Pennington) 1985.

[21] Aoki M., Hanamura S., Masuhara T., Yano K., IEEE Trans. ED 34 (1987) 8.

[22] SElberherR S., IEEE Trans. Electron. Dev. 36 (1989) 1464.

[23] Mathieu H., Physique des Semiconducteurs et des Composants Electroniques, Ed. Masson (Paris) 1987.

[24] Marty A., Rey G., Bailbe J. P., Solid State Electron. 22 (1979) 549.

[25] ReY G., Leturq Ph., Théorie approfondie du transistor bipolaire, Ed. Masson (Paris) 1972.

[26] Jaeger R. C., Gaensslen F. H., IEEE J. Solid State Circ. sc 14 (1979) 423.

[27] Yeargan J. R., Taylor H. L., J. Appl. Phys. 39 (1968) 5600.

[28] Ghibaudo G., J. Phys. C, Solid State Phys. 19 (1985) 767.

[29] Ghibaudo G., Balestra F., Solid State Electron. 32 (1989) 221.

[30] Balestra F., Hafez I. M., Ghibaudo G., ESSDeRC 88, Montpellier, in J. Phys. France 49 (1988) C4-817.

[31] Kelly M. J., The Physics and Fabrication of Microstructures and Microdevices, Ed. M. J. Kelly and C. Wéisbuch (Springer-Verlag, Berlin), 1986, 174.

[32] Peper M., op. cit., p. 323.

[33] Cabon B., Ghibaudo G., Cristoloveanu S., Electron. Lett. 21 (1985) 457.

[34] Nguyen-Duc C., Cristoloveanu S., Ghibaudo G., Solid State Electron. 29 (1986) 1271.

[35] Dierickx B., Warmerdam L., Simoen E., Vermeiren J., Claeys C., IEeE Trans. Electron. Dev. ED-35 (1988) 1120.

[36] Kato K:, Wada T., Tanigushi K., IEEE Trans. Electron. Dev. ED-32 (1985) 458.

[37] Colinge J. P., IEEE Electron. Dev. Lett. EDL-9 (1988) 97.

[38] Balestra F., Ghibaudo G., Solid State Electron. 30 (1987) 321.

[39] Tihanyi J., SchlotTerer H., Solid State Electron. 18 (1975) 305.

[40] IPRI C., Silicon Integrated Circuits, Ed. D. Kahng (Academic Press, New-York) 1981.

[41] BaixerAS J., Rapport de conjoncture et de prospective sur les supraconducteurs à haute température, CNRS-SPI (Paris) 1988.

[42] Gallagher W. J., Solid State Techn. (Nov. 1989) 151.

[43] Flechet B., ChIlo J., Les interconnexions supraconductrices à , haute température, ENSERG/INPG, Rapport (1989).

[44] KeYworth G. A:, MRS Bull (Nov. 1989) 12.

[45] Weber S., Electronics (Sept. 1988) 105. 